As for the evaluation of subjects after ascent by car to a height of $4000 \mathrm{~m}$, a recent survey by Onopa ${ }^{6}$ shows that close to $30 \%$ of day visitors to the Mauna Kea peak in Hawaii experienced acute mountain sickness. One and a half percent had severe illness (poorly responsive on descent). Given the uncertain effect of acute mountain sickness on ABI, the considerable risks involved, and the uncertain role of physical activity on acclimatization, we believe it would be ethically questionable to expose subjects to the risks of ascent by car for the purpose of obtaining data that would most likely be inconclusive.

Patrice Nault, MD, RVT

Gatineau, Quebec, Canada

\title{
References
}

\section{In Reply to Dr Jamshidi's Letter}

\section{To the Editor:}

In response to the letter by Dr Jamshidi, we believe there are sufficient data published to date that describe the effects of physical activity, including strenuous physical activity, on ankle-brachial index (ABI). De Cossard et al, ${ }^{1}$ Abraham et al, ${ }^{2}$ Desvaux et al, ${ }^{3}$ Abraham et al, ${ }^{4}$ and Taylor et $\mathrm{al}^{5}$ have all shown that ABI is lowered after physical activity. It has also been shown that the effects of physical activity on ABI are short lived. ${ }^{4}$ Given that our subjects were at rest for 1 hour before measurements were taken, we believe we have dissociated the effects of physical activity on our results.

1. De Cossard L, Marples M, Cloherty G, Marcuson RW. The monarch bicycle ergometer in screening arterial patients. Br J Surg. 1982;9:543-544.

2. Abraham P, Desvaux B, Saumet JL. Ankle-brachial index after maximum exercise in treadmill and cycle ergometers in athletes. Clin Physiol. 1998;18:321-326.

3. Desvaux B, Abraham P, Colin D, Leftheriotis G, Saumet JL. Ankle to arm index following maximal exercise in normal subjects and athletes. Med Sci Sports Exerc. 1996;28:836-839.

4. Abraham P, Bickert S, Vielle B, Chevalier JM, Saumet JL. Pressure measurements at rest and after heavy exercise to detect moderate arterial lesions in athletes. J Vasc Surg. 2000;33:721-727.

5. Taylor A, George K. Ankle to brachial pressure index in normal subjects and trained cyclists with exercise induced leg pain. Med Sci Sports Exerc. 2001;33:1862-1867.

6. Onapa J, Haley A, Yeow ME. Survey of acute mountain sickness on Mauna Kea. High Alt Med Biol. 2007;7:200-205. 